\title{
Probability distributions of rain attenuation obtainable with linear combining techniques in space-to-Earth links using time diversity
}

\section{Emilio Matricciani}

Politecnico di Milano, Milan, Italy

Correspondence

Emilio Matricciani, Politecnico di Milano,

Piazza L. da Vinci, 32, Milan, Italy.

Email: emilio.matricciani@polimi.it

\begin{abstract}
Summary
The purpose of this paper is to show how the complementary probability distribution of rain attenuation is drastically changed in the lower rain attenuation range by applying linear combining techniques, namely, equal-gain combining and the maximal-ratio combining, discussed in the historical paper by Brennan in 1959. These combing techniques can also be applied to the Automatic Repeat Request techniques. Defined the instantaneous processing gain and the equivalent attenuation in the 3 cases, we show examples of time series of the various parameters, based on the experimental rain attenuation time series recorded with the ITALSAT $18.7 \mathrm{GHz}$ beacon, in a $37.8^{\circ}$ slant path in Spino d'Adda (Italy). Then, we report long-term complementary probability distribution functions of the instantaneous gain and equivalent attenuation, by simulating rain attenuation time series at 19.7 and $39.4 \mathrm{GHz}$, path elevation angle $35.5^{\circ}$, with the Synthetic Storm Technique, using on-site measured rain rate time series of 10 years, by simulating the ALPHASAT link at Spino d'Adda. Similar results are also found at different frequencies and elevation angles in Tampa (Advanced Communications Technology Satellite, ACTS result test), the Isle of Guam, and Prague. The main conclusions are as follows: (1) As expected, the instantaneous time diversity gain can be large when the delay time is large and rain attenuation is large; (2) scintillation affects time diversity links as the direct links; (3) equal-gain and maximal-ratio combining can add up to $3 \mathrm{~dB}$ to the selection diversity gain when the time diversity gain is very small; and (4) equal-gain and maximal-ratio combining reduce the fraction of time of rain attenuation in an average year to a value less than the probability of exceeding $3 \mathrm{~dB}$ in the link without diversity.
\end{abstract}

\section{KEYWORDS}

$A R Q$, diversity gain, equal-gain combining, equivalent attenuation, Guam, maximal-ratio combining, Prague, processing gain, rain attenuation, selection diversity, Spino d'Adda, Synthetic Storm Technique, Tampa

\section{1 | INTRODUCTION}

First proposed by Fukuchi, ${ }^{1}$ time diversity is a long-studied fade countermeasure to rain attenuation, applicable to satellite communication services that can tolerate long time delay (minutes), such as television and audio broadcasting, data transfer from geostationary satellites. ${ }^{1-17}$ Time diversity can be seen as an evolution of the less-demanding Automatic Repeat Request (ARQ) techniques, ${ }^{18}$ because direct and delayed signals are continuously transmitted with a suitable time delay, while in the classic ARQ techniques, the signal is retransmitted in the same channel 2 or more times until it is correctly received. Time diversity needs a redundant channel, therefore it affects radiofrequency equipment and transmitted power, computer central unit processing power and size of memories, both at the transmitter (satellite) and at the receiver (ground).

Time diversity can also be used in near-Earth and deep space links for which, because of the large propagation delay, non-real-time communication is the only possible mode of operation. In this case, the delayed transmission would be programmed in due advance when bad weather is 
forecast at the Earth ground station during the contact time, and the choice of time delay would depend on spacecraft distance and could be of the order of magnitude of the 1-way or 2-way propagation delay, eg, of the order of 30 minutes for missions near Mars. ${ }^{19,20}$

Other troposphere fading, such as that due to water vapour, oxygen, and clouds, can be considered practically constant during the time scale of a rain event; therefore, time diversity is not effective and cannot be proposed for this kind of fading. Scintillation may benefit from time diversity even for small delay, but the gain obtainable is very small, unless very low elevation angles are considered (ie, long paths in the troposphere), and electrically very small antennas are used.

The most effective application of time diversity is likely at $\mathrm{K}_{\mathrm{a}}$ band and above, both because a wider bandwidth is available and rain attenuation is larger than at lower frequency bands. Its efficacy is based on the experimental evidence that rain attenuation decorrelates after few minutes at large fades, and after few tens of minutes at small fades, so that, by transmitting twice (or more times) the same signal with a suitable fixed time delay of the order of several minutes, the receiver can select the best signal and reduce link outage. This description suits the analysis that propagation experts conducted in the past ${ }^{1-17}$ and known, in the theory of diversity systems, as selection diversity. Only recently linear combining techniques have been applied to time diversity system. ${ }^{21}$

The aim of this paper is to show how the complementary probability distribution function (CDF, also known as exceedance probability distribution) of rain attenuation is drastically changed in the lower attenuation range if linear combining techniques are applied. The results are useful and applicable to future satellite links. Technology issues, some of which are discussed in Uggalla, ${ }^{21}$ are beyond the purpose of this paper.

Besides selection diversity, we consider the other 2 types of processing that are possible with the direct signal $s(t)$ (not delayed) and the $T-$ min delayed signal $s(t-T)$, namely, the equal-gain combining and the maximal-ratio combining, discussed by Brennan in $1959 .{ }^{22}$ These combining techniques can also be applied to the classic $A R Q$ techniques.

After this introduction, Section 2 summarises the theoretical signal-to-noise ratio obtainable with the 3 types of processing; Section 3 defines the processing gain and the equivalent attenuation obtainable in each case; and Section 4 shows time series of the various parameters defined in Section 3, based on the experimental rain attenuation time series recorded in Spino d'Adda from the ITALSAT $18.7 \mathrm{GHz}$ beacon, in a $37.8^{\circ}$ slant path. Section 5 reports long-term statistical results of the parameters at 19.701 and $39.4 \mathrm{GHz}$, path elevation angle $35.5^{\circ}$, obtained with the Synthetic Storm Technique (SST), ${ }^{23}$ using on-site measured rain rate time series (averaged in 1-min intervals, as are also the other rain rate data considered in the paper) for 10 years, simulating the ALPHASAT link in Spino d'Adda. Section 6, to show that linear combining techniques produce similar results also in other sites, first tests the SST in Tampa at $27.5 \mathrm{GHz}$ and then reports time diversity results concerning Tampa, the Isle of Guam, and Prague, as examples. Section 7 reports conclusions and final remarks. Appendix A lists the mathematical symbols and their definition, Appendix B discusses the impact of sky noise on time diversity links, and Appendix $C$ discusses why scintillation affects time diversity links as the direct links.

\section{2 | TIME DIVERSITY AND ARQ WITH COMBINING}

The linear combining techniques discussed in this section can be applied both to time diversity that uses 2 (or more) parallel channels and to the classic ARQ techniques in which retransmission in the unique channel would be requested only if the linear combining of $s(t)$ with $s(t-T)$ does not guarantee the minimum tolerated probability of symbol error. In other words, the linear combining techniques are general so that, in the following, we will not distinguish between the two in the results on rain attenuation. In the following, to fully appreciate the performance of the different combining techniques, we present the rain attenuation CDFs obtainable with the different combining techniques.

Let $A(t)>0$ and $A(t+T)>0$ be the rain attenuation, in $d B$, at the same instants of $s(t)$ ands $(t-T)$, the baseband equivalent signals. Let us set the time origin of the direct link at the beginning of the rain attenuation event, so that $A(t)>0$ for $t>0$. From the point of view of signal processing, we can assume that $s(t)$ and $s(t-T)$ are transmitted in 2 (nonsimultaneous) parallel radio links, referred to as the diversity link. The corresponding baseband noise sources $n(t)$ and $n(t+T)$ are modelled as additive and independent Gaussian variables, with one-sided power spectral density $N_{0}$. Before deciding which symbol was transmitted, the diversity receiver can process the 2 continuous baseband signals in 3 (classical) ways ${ }^{19}$ :

\section{Selection diversity}

2. Equal-gain diversity combining.

3. Maximal-ratio diversity combining. Case 2 is a special case of case 3 when the 2 signals are equally weighted before combining.

In selection diversity, the receiver can compare the output power of the 2 channels and select the channel with maximum power, which implies, for constant noise power (discussed in Appendix B), that it compares $s(t)$ with $s(t+T)$ and thus selects the largest signal. Therefore, the diversity link is faded by the minimum rain attenuation $A_{m}(\tau)=\min \{A(t), A(t+T)\}$, with $\tau=t+T$.

The variable $A_{m}$ is attributed to the instant $\tau=t+T>0$ because the selection (or any combining and decision) can only be done, of course, after $T$ minutes. Therefore, $\tau$ is the time axis of the diversity link. Moreover, during the ith rain attenuation event of duration $D_{i}$ (min) (namely, a rain attenuation time series), the last selection (or combining) can only be done at $t=D_{i}-T$ because, fort $>D_{i}-T, A(\tau)=0$. Therefore, for each rain event, the time diversity link measures a total rain attenuation time $T$ minutes shorter than of the direct link; hence, for selection diversity, the fraction of time, in an average observation period such as a year, $P_{0, m}=P\left(A_{m}>0\right)<P_{0}=P(A>0)$, where $P\left(A_{m}\right)$ and $P(A)$ are, respectively, the CDFs referred to an average year, in the diversity link and in the direct link. 
The instantaneous time diversity gain, $G_{T}(\tau)(d B)$, that the selection diversity can theoretically achieve is given by

$$
G_{T}(\tau)=A_{\max }(\tau)-A_{m}(\tau),
$$

where $A_{\max }=\max [A(t), A(\tau)]$ is the largest of the two.

The instantaneous signal-to-noise ratios of the direct link, $\rho(t)$, and of the diversity link with selection diversity, $\rho_{S}(\tau)$, are given (matched filter receivers) by

$$
\begin{gathered}
\rho(t)=\frac{E_{s}}{N_{o} / 2}=\frac{P_{r}}{N_{o} R / 2}=\frac{P_{r, c s} \times 10^{-A(t) / 10}}{N_{o} R / 2}, \\
\rho_{S}(\tau)=\frac{E_{s, m}}{N_{o} / 2}=\frac{P_{r, m}}{N_{o} R / 2}=\frac{P_{r, c s} \times 10^{-A_{m}(\tau) / 10}}{N_{o} R / 2} .
\end{gathered}
$$

In Equations $2 \mathrm{a}$ and $2 \mathrm{~b}, R$ is the symbol rate, $E_{s}$ is the received energy per symbol of $s(t), P_{r}$ is the carrier average power, $E_{s, m}$ is the energy per symbol of $\max [s(t), s(\tau)], P_{r, m}$ is the carrier average power, $P_{r, c s}$ is the carrier average power in clear sky, and $N_{o}$ is the one-sided noise power spectral density. Any coding gain can be, of course, included in Equations $2 \mathrm{a}$ and $2 \mathrm{~b}$, without changing time diversity results.

In equal-gain diversity combining, after $T$ minutes, the receiver adds the 2 total amplitudes (signal + noise) after phasing them, therefore adding linearly the amplitudes of the 2 (theoretically synchronised) signals and the power of the 2 independent noise sources, doubling (see Appendix B) the one-sided power spectral density. Hence, the signal-to-noise ratio, $\rho_{E}(\tau)$, is given by

$$
\begin{aligned}
& \rho_{E}(\tau)=\frac{P_{r, c s} \times\left\{10^{-A(t) / 20}+10^{-A(\tau) / 20}\right\}^{2}}{\left(2 N_{o}\right) R / 2}= \\
& \rho_{E}(\tau)=\frac{1}{2}\left\{\frac{P_{r, c s} \times 10^{-A(t) / 10}}{N_{o} R / 2}+\frac{P_{r, c s} \times 10^{-A(\tau) / 10}}{N_{o} R / 2}\right\}+\frac{P_{r, c s} \times 10^{-}}{N_{o} R / 2}
\end{aligned}
$$

Expression 3 is the average of the 2 signal-to-noise ratios (Equation 2a) calculated at $t$ and $\tau$, added to the signal-to-noise ratio (Equation 2a) due to the average rain attenuation.

In maximal-ratio diversity combining, after $T$ minutes, the receiver adds the 2 total amplitudes, as with equal-gain diversity combining, but weighting each with the square root of its own signal-to-noise ratio. Theoretically, this combining delivers the maximum signal-to-noise ratio, given by the sum of the signal-to-noise ratios of the 2 parallel links 22 :

$$
\rho_{M}(\tau)=\rho(t)+\rho_{S}(\tau) .
$$

Let us now compare the 3 linear diversity techniques and define the processing gain and the equivalent rain attenuation, both parameters useful for link design.

\section{3 | PROCESSING GAIN AND EQUIVALENT RAIN ATTENUATION}

We can assess the efficacy of equal-gain and maximal-ratio combining by referring their gain to that of selection diversity. For this purpose, let us define the processing gain as the ratio between Equations 3 and 2b, or Equations 4 and 2b.

For equal-gain diversity combining, the processing gain $g_{E}(\tau)$ is given by:

$$
g_{E}(\tau)=\frac{\rho_{E}(\tau)}{\rho_{S}(\tau)}=\frac{1}{2}\left(1+10^{-\frac{G_{T}(\tau)}{20}}\right)^{2} \quad 0.5 \leq g_{E} \leq 2
$$

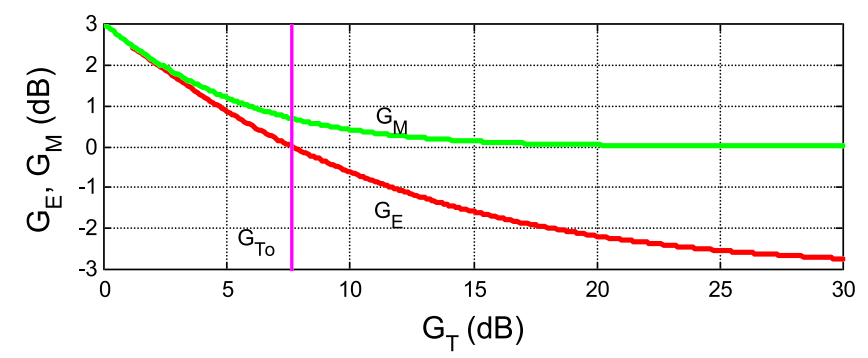

FIGURE 1 Equal-gain combining gain $G_{E}$ (red line) and maximal-ratio combining gain $G_{M}$ (green line) versus time diversity gain $G_{T}$. The vertical (magenta) line gives $G_{T o}$, which sets the range $G_{T}<G_{T o}$ in which it is convenient to use equal gain combining, see Equation 8 [Colour figure can be viewed at wileyonlinelibrary.com] 
For maximal-ratio diversity combining,

$$
g_{M}(\tau)=\frac{\rho_{M}(\tau)}{\rho_{S}(\tau)}=1+10^{-\frac{G_{T}(\tau)}{10}} \quad 1 \leq g_{M} \leq 2
$$

If expressed in $\mathrm{dB}, G_{E}=10 \times \log _{10}\left(g_{E}\right)$ and $G_{M}=10 \times \log _{10}\left(g_{M}\right)$ give the further gain that the particular combining can add to the time diversity gain $G_{T}(\tau)$, therefore providing the total instantaneous gain $G_{t}(\tau)(d B)$ :
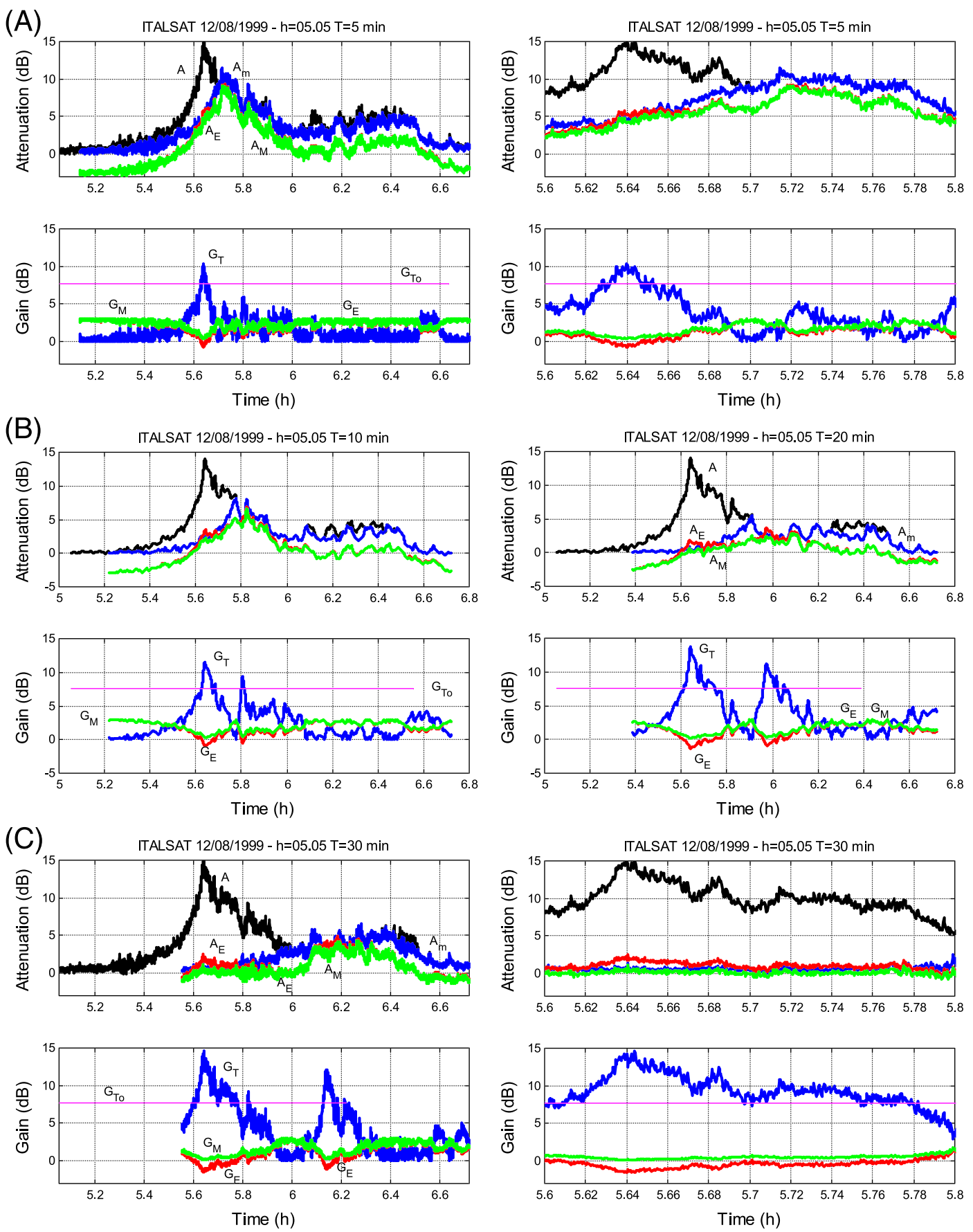

FIGURE 2 A, Example of rain attenuation time series with scintillation at $18.7 \mathrm{GHz}, 38.8^{\circ}$ slant path, $T=5$ minutes. Left: Upper figure, $A(t)$ (black), $A_{m}(\tau)$ (blue), $A_{E}(\tau)$ (red), and $A_{M}(\tau)$ (green); lower figure, $G_{T}(\tau)$ (blue), $G_{E}(\tau)$ (red), and $G_{M}(\tau)$ (green). The horizontal (magenta) line gives $G_{T o}$, which sets the range $G_{T}<G_{T o}$ in which it is convenient to use equal-gain combining, see Equation 8. Right: Detail to show that scintillation amplitude is insensitive to combining. Local time. B, Rain attenuation time series without scintillation (filtered with a fifth-order Butterworth filter with cutoff frequency $0.025 \mathrm{~Hz}$ ). Left: $T=10$ minutes. Upper figure, $A(t)$ (black), $A_{m}(\tau)$ (blue), $A_{E}(\tau)$ (red), and $A_{M}(\tau)$ (green); lower figure, $G_{T}(\tau)$ (blue), $G_{E}(\tau)$ (red), and $G_{M}(\tau)$ (green). The horizontal (magenta) line gives $G_{T o}$, which sets the range $G_{T}<G_{T_{o}}$ in which it is convenient to use equal-gain combining, see Equation 8. Right: Same, but for $T=20$ minutes. Local time. C, Rain attenuation time series with scintillation. $T=30$ minutes. Upper figure, $A(t)$ (black), $A_{m}(\tau)$ (blue), $A_{E}(\tau)$ (red), and $A_{M}(\tau)$ (green); lower figure, $G_{T}(\tau)$ (blue), $G_{E}(\tau)$ (red), and $G_{M}(\tau)$ (green). The horizontal (magenta) line gives $G_{T o}$, which sets the range $G_{T}<G_{T o}$ in which it is convenient to use equal-gain combining, see Equation 8. Right: Detail to show that scintillation amplitude is insensitive to combining. Local time [Colour figure can be viewed at wileyonlinelibrary.com] 


$$
\begin{aligned}
& G_{t E}(\tau)=G_{T}(\tau)+G_{E}(\tau), \\
& G_{t M}(\tau)=G_{T}(\tau)+G_{M}(\tau) .
\end{aligned}
$$

From Equation 5, $G_{E}$ can be either positive or negative; from Equation 6, $G_{M}$ is only positive, therefore $G_{t M} \geq G_{T}$ whereas $G_{t E}>G_{T}$ or $G_{t E}<G_{T}$. Figure 1 shows $G_{E}$ and $G_{M}$ versus $G_{T}$. We can notice that $G_{E} \geq 0 \mathrm{~dB}$ in the range

$$
G_{T} \leq G_{T o}=-20 \log _{10}(\sqrt{2}-1) \approx 7.7 \mathrm{~dB} \text {. }
$$

Therefore, it is convenient to use equal-gain combining only if $G_{T}<G_{T o}$.

As Figure 1 shows, $G_{E}$ and $G_{M}$ are maximum and equal to the built-in power margin $G_{b}=10 \times \log _{10} n=3 \mathrm{~dB}(\mathrm{n}=2$ is the number of parallel links combined, see Matricciani ${ }^{24}$ for its application to site diversity) found any time $G_{T}=0 \mathrm{~dB}$, therefore when $A(t)=A(t+T)$. Moreover, if $G_{T} \rightarrow \infty$, then $G_{E} \rightarrow-3 \mathrm{~dB}$ (a loss, not a gain), and $G_{M} \rightarrow 0 \mathrm{~dB}$, because the maximal-ratio combining gives always a positive gain.

Because $G_{T} \approx 0$ is more probable to occur when rain attenuation is small, we get an almost "free" gain to add to $G_{T}$ when it is most needed, namely, when time diversity is ineffective. This can happen for long intervals and a large total amount of time, because a small attenuation is more probable than a large one. When $G_{T} \approx 0$, however, the gain due to equal-gain combining is practically the same as that due to maximal-ratio combining, so that it might be preferable to use the simpler equal-gain combining.

The built-in power margin (also known as the array power gain), $G_{b}$, is independent of frequency, therefore, in general, combining is more effective at lower frequency bands than at higher ones, because, everything else being equal (slant path, probability, etc), at lower carrier frequencies, rain attenuation is lower and hence $G_{b}$ has more impact on system design than at higher frequencies.

Let us know define the equivalent rain attenuation of the diversity link, that is, the attenuation that has to be considered in the link budget.

In selection diversity, the equivalent rain attenuation $A_{m}(\tau)(d B)$ from Equation 1 is given by

$$
A_{m}(\tau)=A_{\max }(\tau)-G_{T}(\tau)
$$
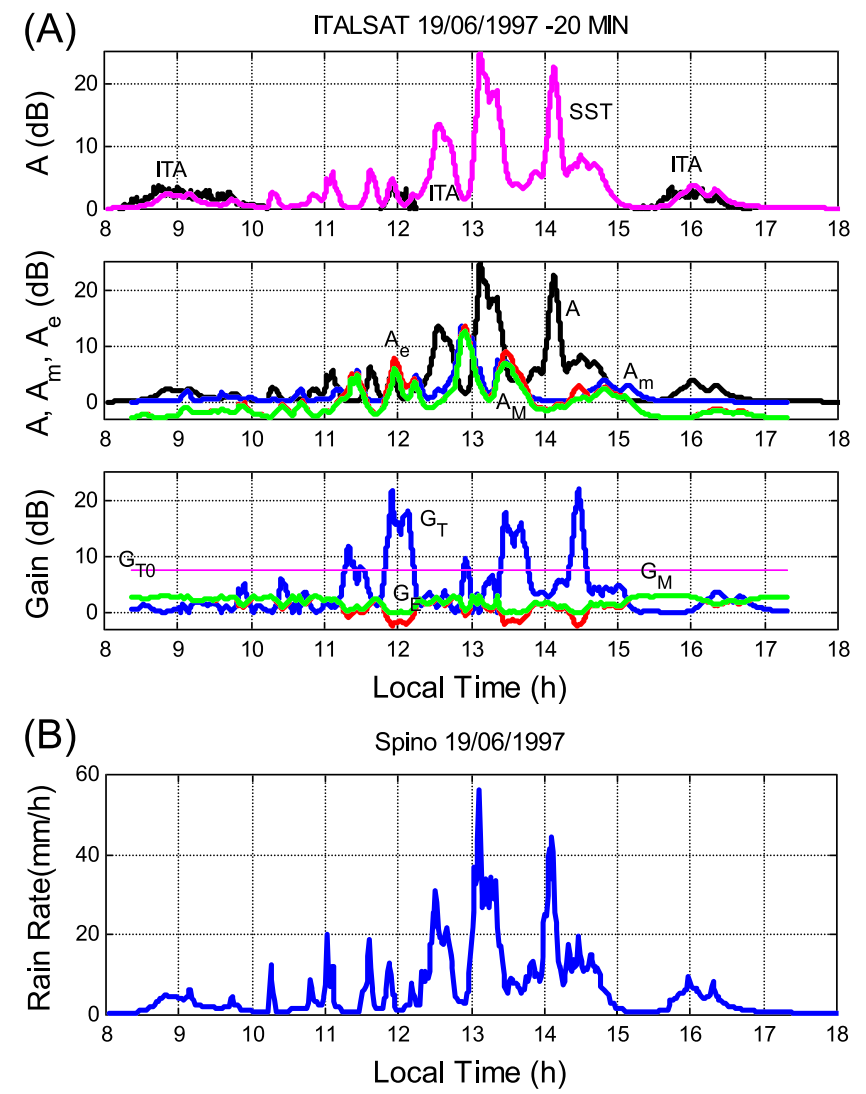

FIGURE 3 A, Upper figure: An example of partial rain attenuation time series at $18.7 \mathrm{GHz}$ measured during the ITALSAT campaign at Spino d'Adda, in a $37.8^{\circ}$ slant path (black line). Most of the event is , missing but it can be reconstructed (magenta line) very likely with the Synthetic Storm Technique (SST) using, as input, the concurrent rain rate time series measured at the station. Middle figure: $A(t)$ (black), $A_{m}(\tau)$ (blue), $A_{E}(\tau)$ (red), and $A_{M}(\tau)$ (green) simulated with the SST, for $T=20$ minutes, in the same link at $18.7 \mathrm{GHz}$. Lower figure: $G_{T}(\tau)$ (blue), $G_{E}(\tau)$ (red), and $G_{M}(\tau)$ (green) simulated with the SST. The horizontal (magenta) line gives $G_{T o}$, which sets the range $G_{T}<G_{T o}$ in which it is convenient to use equal-gain combining, see Equation 8. B, Rain rate time series of the event occurred in Spino d'Adda on June 19, 1997 [Colour figure can be viewed at wileyonlinelibrary.com] 
In equal-gain and maximal-ratio combining, the equivalent rain attenuation, $A_{E}(\tau)$ and $A_{M}(\tau)(d B)$, respectively, is defined with reference to $A_{m}(\tau)$, as

$$
\begin{aligned}
& A_{E}(\tau)=A_{m}(\tau)-G_{E}(\tau), \\
& A_{M}(\tau)=A_{m}(\tau)-G_{M}(\tau) .
\end{aligned}
$$

Because $G_{E}(\tau)$ can be negative, we can find $A_{E}(\tau)>A_{m}(\tau)$, occurrence to be avoided.

Next section shows some examples of rain attenuation time series of $A(t), A_{m}(\tau), A_{E}(\tau), A_{M}(\tau), G_{T}(\tau), G_{E}(\tau)$, and $G_{M}(\tau)$, based on the experimental rain attenuation time series recorded from the ITALSAT $\left(13.2^{\circ} \mathrm{E}\right) 18.7 \mathrm{GHz}$ beacon, in a $37.8^{\circ}$ slant path in Spino $\mathrm{d}^{\prime} \mathrm{Adda}\left(45.4^{\circ} \mathrm{N}, 9.5^{\circ} \mathrm{E}, 180 \mathrm{~m}\right.$ ) sampling time 1 second, with different values of time delay $T$, although the conclusions are general.

\section{4 | EXAMPLES OF ITALSAT TIME SERIES AT $18.7 \mathrm{GHz}$}

Figure 2 shows examples of $A(t), A_{m}(\tau), A_{E}(\tau), A_{M}(\tau), G_{T}(\tau), G_{E}(\tau)$, and $G_{M}(\tau)$, concerning a rain attenuation event with large attenuation, with scintillation and without scintillation (the latter filtered out with a fifth-order Butterworth filter, cutoff frequency $0.025 \mathrm{~Hz}$, see $\mathrm{Matricciani}$ and $\operatorname{Riva}^{25}$ ). We can see the intervals when combining is effective, mainly when the attenuation is small, and when it is less effective, when the attenuation is large.

The right side of Figure $2 A(T=5 \mathrm{~min})$ shows a detail where we can notice that the scintillation in $A_{E}(\tau)$ and in $A_{M}(\tau)$ is not larger than that found in $A_{m}(\tau)$. This behaviour is discussed in Appendix $C$ and leads to the conclusion that combining does not increase the scintillation of the diversity link, compared to that of the direct link. Now, time diversity is not affected by scintillation because the gain due to scintillation is very small and can be neglected for system design. Therefore, we can use filtered rain attenuation time series, or times series simulated with the
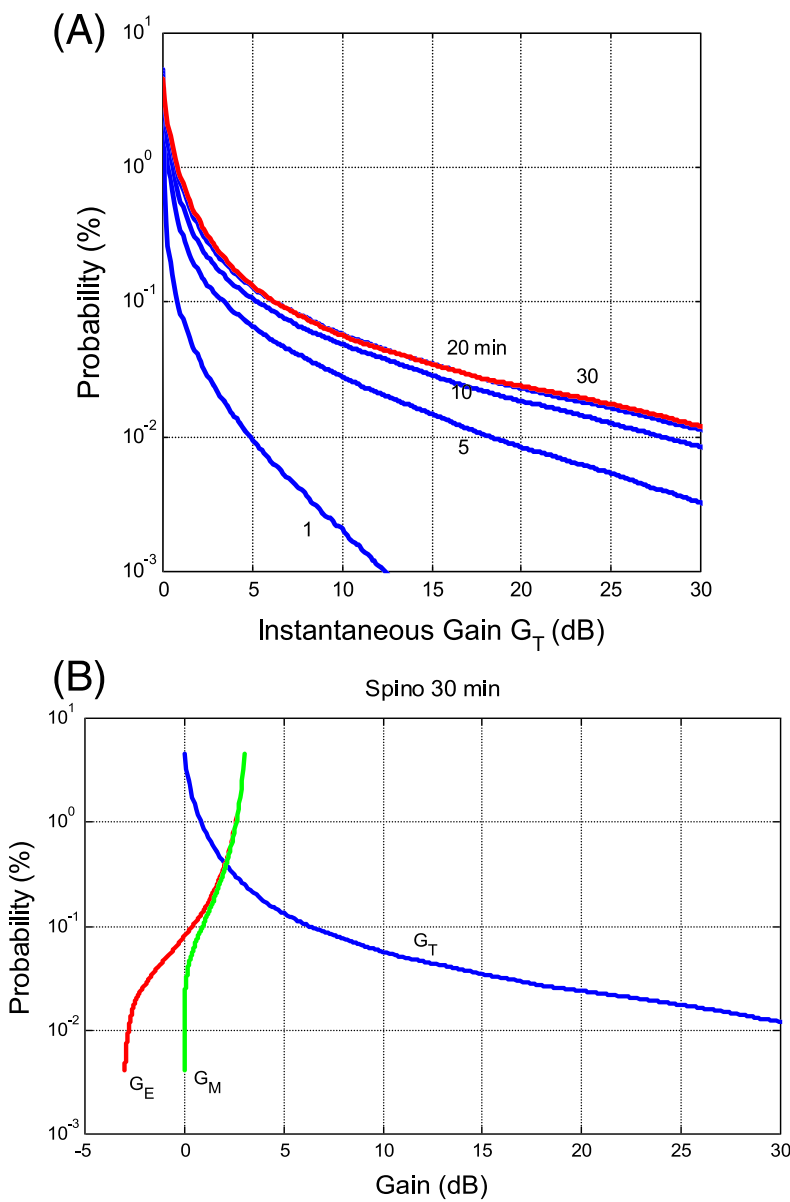

FIGURE 4 A, Complementary probability distribution functions, in an average year (\%), of the instantaneous gain $G_{T}(\mathrm{~dB})$ for time delay $T=1$, 5 , 10, 20 minutes (blue) and $T=30$ minutes (red). Saturation occurs at $T \sim 20,30$ minutes. Synthetic Storm Technique. B, Complementary probability distribution function, in an average year (\%), of the instantaneous gain $G_{T}$ (blue) and the probability distribution functions of $G_{E}$ (red) and $G_{M}$ (green) for $T=30$ minutes. For $G_{E}$ and $G_{M}$, the functions give the probability that the variable is less than that reported in the abscissa. Synthetic Storm Technique [Colour figure can be viewed at wileyonlinelibrary.com] 
SST without adding scintillation, for studying the performance of time diversity and combining. Figure $2 \mathrm{~B}$ shows the filtered time series for $T=10,20$ minutes.

In Figure 2C, T= 30 minutes, we can also observe time diversity in action. In this event, the 4-dB threshold, for instance, is exceed continuously for about 30 minutes (fade duration) in the direct link, ie, in $A(t)$, and accordingly, $A_{M}(\tau)$ and $A_{E}(\tau)$ never exceed $4 \mathrm{~dB}$ because $T=30$ minutes. In other words, considered a threshold $S=A(\mathrm{~dB})$, its maximum fade duration sets the delay time $T$ to be used for never exceeding the threshold $S$. In system design, there must be, of course, a trade-off between the time delay introduced in the link and the maximum fade tolerated, on the average, in the service area, in case of many ground stations. In general, the longer is $T$, the lower are $S$ and the link power margin needed, but the more demanding are hardware (memories) and software (signal processing) to be implemented. These issues are not considered here.

The ITALSAT rain attenuation times series are very useful for studying time-varying aspects of the fade (not only rain attenuation). However, for rain attenuation, they are not a complete set in the 7 years of observation, as we observed, ${ }^{26}$ because some events were totally or partially missed because of receiver malfunctioning. An example is shown in Figure 3A, which reports also the time series predicted with the SST, 22 by using the concurrent rain rate time series measured at the station and shown in Figure 3B, and the time diversity performance for $T=20$ minutes. The simulated time series seems to describe very likely what was missed. In general, as we have shown in Matricciani, ${ }^{27}$ a beacon-measured time series and the corresponding SST time series are very similar when the storm moves parallel to the projection to ground of the slant path. In all other cases, the direction and speed of the rain introduce delays and "deformation" in the simulated time series, ${ }^{27}$ which, however, remain physically likely for the site and can be used for long-term results.

Notice that Capsoni et al assume synthetic rain rate cells of deterministic shape with rain rate established by assigning random parameters to the cell (peak, extension, etc) and make them move horizontally. ${ }^{12}$ Then, in a slant path, they calculate the attenuation as line integral of the specific attenuation based on the intercepted cells. The random parameters of the rain rate cells are chosen so that the local long-term complementary probability distribution of rain rate is maintained. The synthetic rain attenuation time series so generated very unlikely can resemble the one recorded with raingauge.

Because of this incompleteness, the next section presents long-term results obtained by using 10 years of rain rate time series (concurrent with the ITALSAT beacon data in the 7 years of this campaign) recorded at Spino d'Adda and used as input to the SST.
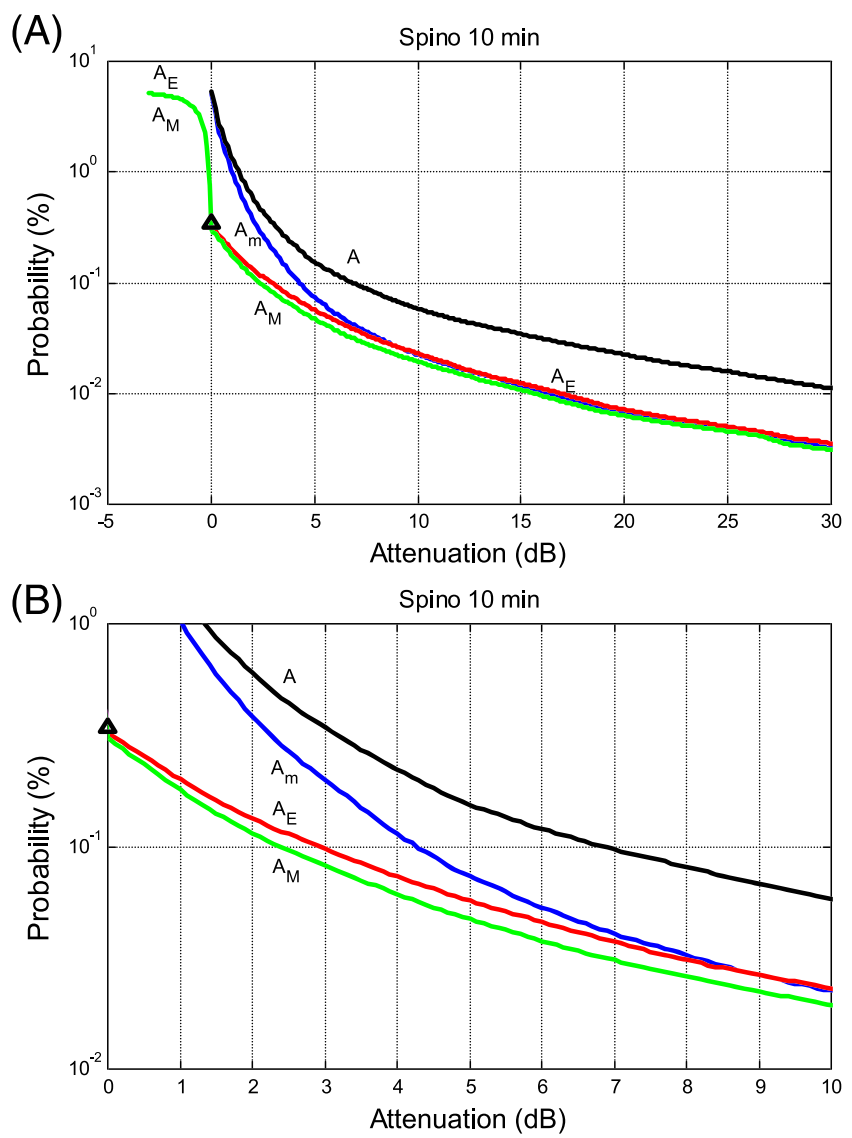

FIGURE 5 A, Complementary probability distribution functions, in an average year (\%), of $A$ (black), $A_{m}$ (blue), $A_{E}$ (red), and $A_{M}$ (green), for $T=10$ minutes. In the range $-3,0 \mathrm{~dB}, A_{E}$ and $A_{M}$ are very similar. The triangle gives $P(A \geq 3)$, that is, the probability that the rain attenuation equal to the built-in power gain is exceeded in the direct link (black line). Synthetic Storm Technique. B, Detail of Figure 5A [Colour figure can be viewed at wileyonlinelibrary.com] 


\section{5 | LONG-TERM COMPLEMENTARY PROBABILITY DISTRIBUTIONS ACCORDING TO THE SST}

For obtaining long-term statistics of time diversity performance, namely, the results concerning $A, A_{m}, A_{E}, A_{M}, G_{T}, G_{E}$, and $G_{M}, I$ use what is practically a complete data bank of rain rate time series (10 years), recorded at the station of Spino d'Adda, used as input to the SST, with the following input parameters: rain speed $10.6 \mathrm{~m} / \mathrm{s}$ measured locally, layer A (rain) height given by the current ITU-R $0^{\circ} \mathrm{C}$ height, ${ }^{28}$ and layer $\mathrm{B}$ (melting layer) height 400 m. ${ }^{23,29}$ The simulations are at 19.7 and $39.4 \mathrm{GHz}$ in a $35.5^{\circ}$ slant path because now they explicitly refer to the radio link to ALPHASAT, and they are likely to predict results very similar to the measurements, now under way in Spino d'Adda.

Figure 4A shows the probability density function of the instantaneous gain $G_{T}$, for $T=1,5,10,20,30$ minutes. These delays can be reasonable for system application, but the practical value (even larger than those indicated) will depend on many compromises between the final user's necessities in the service area, or deep space probe distance, and the technology available, as mentioned in Section 4. Gain saturation occurs at $T \sim 20,30$ minutes. After the discussion in Section 2, the fraction of attenuation time, and also of $G_{T}$, is given by $P_{0, m}<P_{0}$. Recall that these results are always based on being both $A(t)>0$ and $A(t+T)>0$ so that the diversity link rain attenuation for each rain event lasts $T$ minutes less than the direct link. Figure $4 B$ compares the results on $G_{T}, G_{E}$, and $G_{M}$ for $T=30$ minutes. For $G_{E}$ and $G_{M}$, the curves give the probability that the variable is less than that reported in the abscissa.

Figure $5 \mathrm{~A}$ shows the complementary probability distributions functions of $A, A_{m}, A_{E}$, and $A_{M}$ for $T=10$ minutes. Now, if we consider the real loss, namely, the positive range shown in Figure 5A, we find the following inequalities, which are important for system design:

$$
P_{0, E}=P\left(A_{E}>0\right)=P_{0, M}=P\left(A_{M}>0\right)<<P\left(A_{m}>0\right)<P(A>0) .
$$

Of course,

$$
P\left(A_{E}>-3\right)=P\left(A_{M}>-3\right)=P\left(A_{m}>0\right)<P(A>0) .
$$

Figure 5B shows the details in the lower positive range to appreciate the reduction of the equivalent attenuation due to combining. The inequalities $10 \mathrm{a}, 10 \mathrm{~b}$, and $10 \mathrm{c}$ are more verified as the time delay $T$ increases.
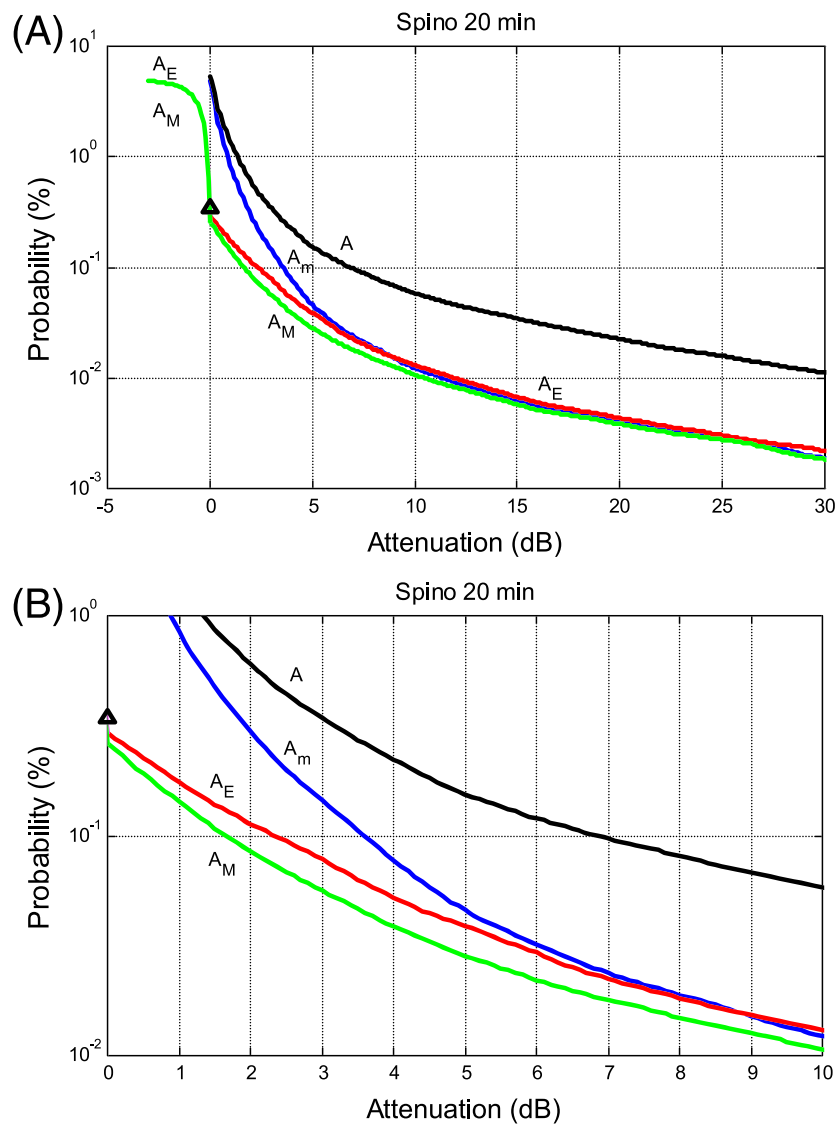

FIGURE 6 A, Complementary probability distribution functions, in an average year (\%), of $A$ (black), $A_{m}$ (blue), $A_{E}\left(\right.$ red), and $A_{M}$ (green), for $T=20 \mathrm{~min}$. In the range $-3,0 \mathrm{~dB}, A_{E}$ and $A_{M}$ are very similar. The triangle gives $P(A \geq 3)$, that is, the probability that the rain attenuation equal to the built-in power gain is exceeded in the direct link (black line). Synthetic Storm Technique. B, Detail of Figure 6A [Colour figure can be viewed at wileyonlinelibrary.com] 
In Spino d'Adda in a year, on the average, we count 1412/10 = 141.2 rain events (see Matricciani ${ }^{23}$ for the definition of a rain event); therefore the fraction of rain attenuation, for $T=30$ minutes, changes from 470.89 hours $\left(P_{0}=5.38 \%\right)$ to $470.89-141.2 \times 0.5=400.29$ hours, therefore the reduction is $400.29 / 470.89=0.85$ and $P_{0, m}=0.85 \times 5.376=4.57 \%$.

Similar results are shown in Figures 6 and 7 for $T=20$ and $T=30$ minutes, respectively, from which we can appreciate the sensitivity to changes in time delay. Figure 7C, in particular, shows the negative range to better distinguish between $A_{E}$ and $A_{M}$.

The statistical gain usually reported in the literature, namely, the gain that can be calculated in Figures $5-7$ by considering the difference between the rain attenuation exceeded in the direct link and that exceeded in the diversity link at the same probability, may be less precise for link design than the instantaneous gain calculated from its probability density function reported in Figure 4.

Equal-gain and maximal-ratio combining reduce the equivalent attenuation at most by $3 \mathrm{~dB}$ (the built-in gain), mainly when $G_{T}$ is small. But more important, they also largely reduce the fraction of rain attenuation time in an average year (or any other reference period). In fact, the triangle shown in Figures 5-7 for $A_{E}=A_{M}=0$ gives $P(A \geq 3)$, that is, the probability that the rain attenuation equal to the built-in power gain is exceeded in the direct link. This probability is the upper value to the fraction of rain attenuation of the diversity link with equal-gain or maximal-ratio combing,
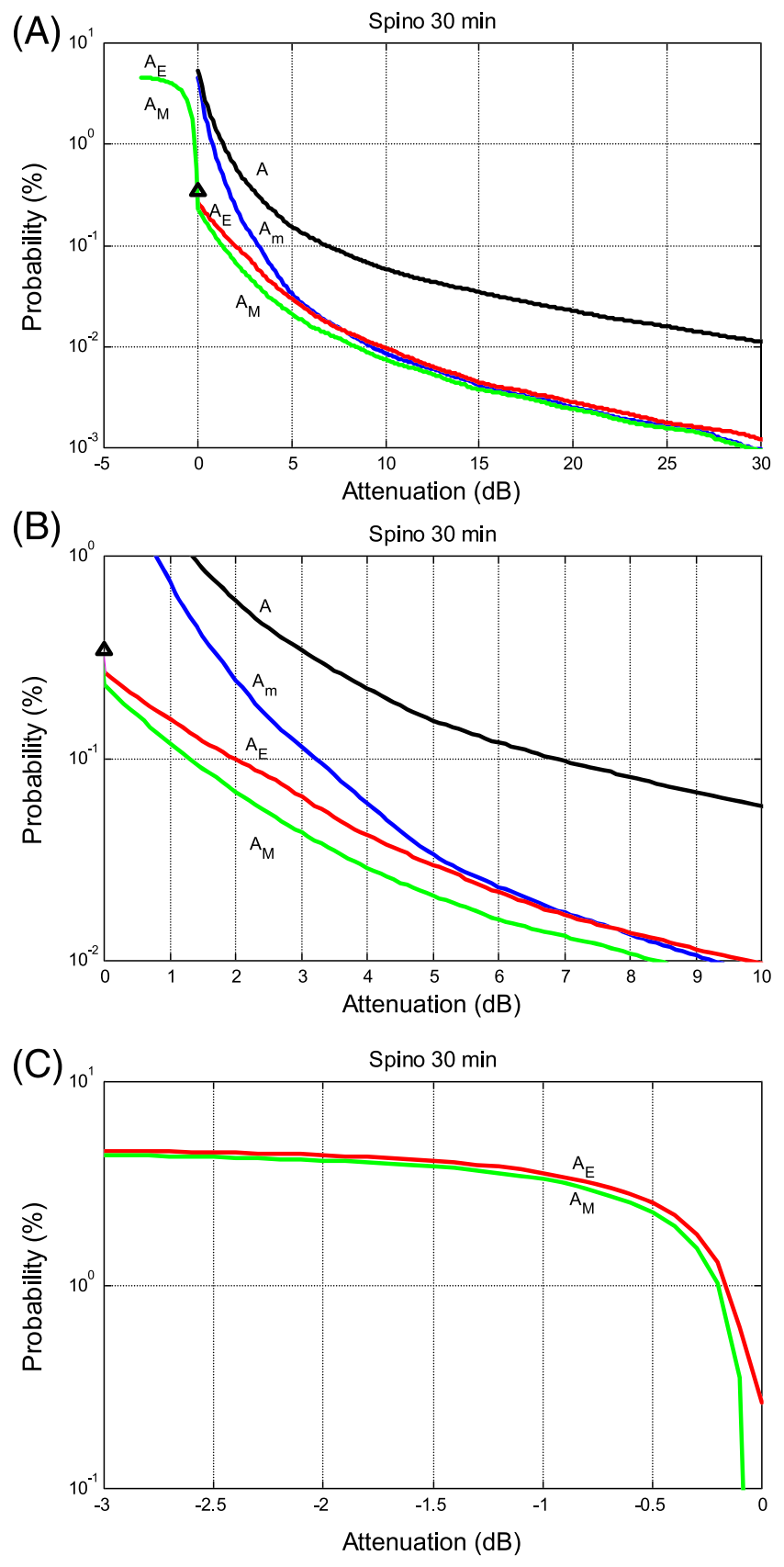

FIGURE 7 A, Complementary probability distribution functions, in an average year (\%), of $A$ (black), $A_{m}$ (blue), $A_{E}$ (red), and $A_{M}$ (green), for $T=30$ minutes. In the range $-3,0 \mathrm{~dB}, A_{E}$ and $A_{M}$ are very similar. The triangle gives $P(A \geq 3)$, that is, the probability that the rain attenuation equal to the built-in power gain is exceeded in the direct link (black line). Synthetic Storm Technique. B, Detail of Figure 7A. C, Detail of Figure 7A [Colour figure can be viewed at wileyonlinelibrary.com] 


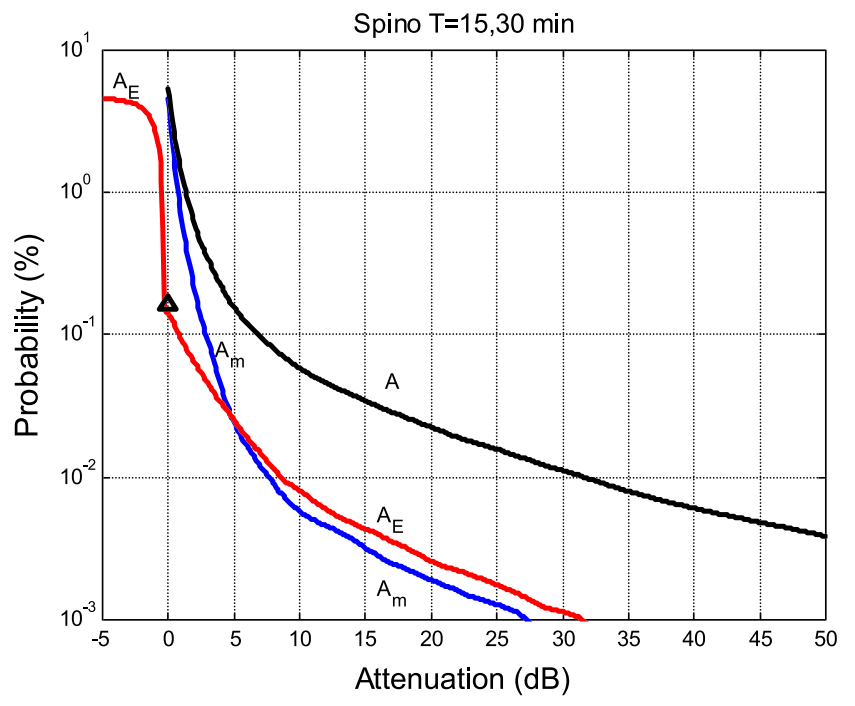

FIGURE 8 Complementary probability distribution functions, in an average year (\%), of $A$ (black), $A_{m}$ (blue), and $A_{E}$ (red), for $T_{1}=15$ and $T=30$ minutes. The triangle gives $P(A \geq 4.8)$, that is, the probability that the rain attenuation equal to the built-in power gain is exceeded in the direct link (black line). Synthetic Storm Technique [Colour figure can be viewed at wileyonlinelibrary.com]

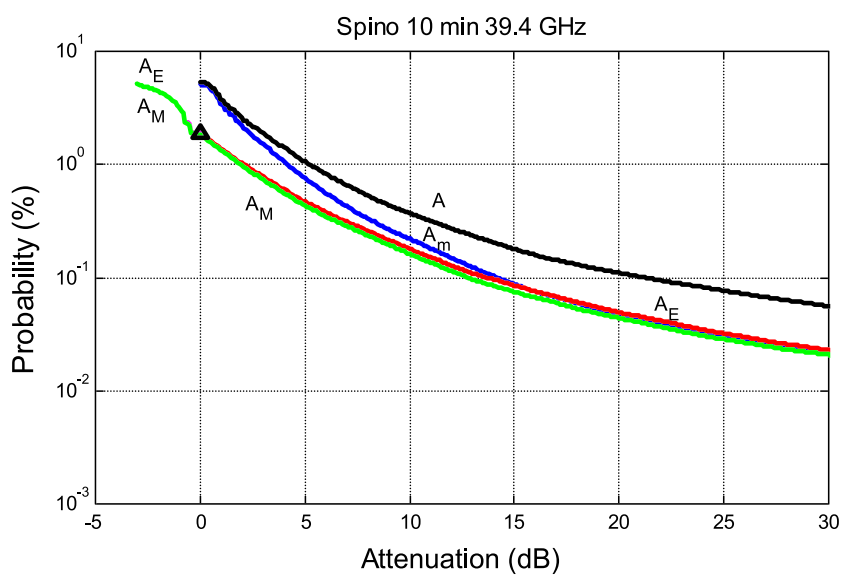

FIGURE 9 Complementary probability distribution functions, in an average year (\%), of $A$ (black), $A_{m}$ (blue), $A_{E}$ (red), and $A_{M}$ (green), at $39.4 \mathrm{GHz}$, for $T=10 \mathrm{~min}$. In the range $-3,0 \mathrm{~dB}, A_{E}$ and $A_{M}$ are very similar. The triangle gives $P(A \geq 3)$, that is, the probability that the rain attenuation equal to the built-in power gain is exceeded in the direct link (black line). Synthetic Storm Technique [Colour figure can be viewed at wileyonlinelibrary.com]

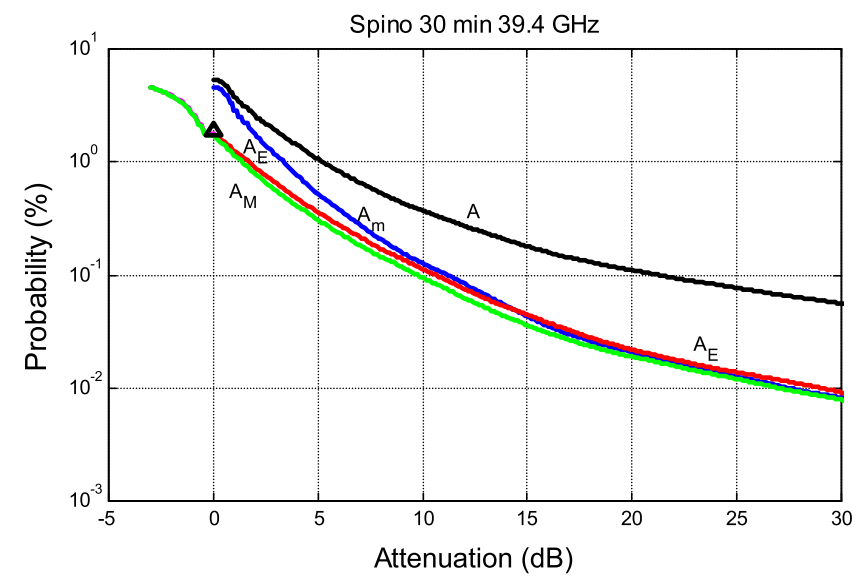

FIGURE 10 Complementary probability distribution functions, in an average year (\%), of $A$ (black), $A_{m}$ (blue), $A_{E}$ (red), and $A_{M}$ (green), at $39.4 \mathrm{GHz}$, for $T=30$ minutes. In the range $-3,0 \mathrm{~dB}, A_{E}$ and $A_{M}$ are very similar. The triangle gives $P(A \geq 3)$, that is, the probability that the rain attenuation equal to the built-in power gain is exceeded in the direct link (black line). Synthetic Storm Technique [Colour figure can be viewed at wileyonlinelibrary.com] 
because, besides subtracting $3 \mathrm{~dB}$ from $A_{m}$, for each rain attenuation time series, as noticed, the diversity link time series lasts $T$ minutes less than that of the direct link. In other words,

$$
P_{0, E}=P_{0, M}<P\left(A>10 \times \log _{10} n\right)
$$

Again, this inequality is more verified as $T$ increases.

In triple time diversity, selection does not reduce $G_{T}$ significantly, compared to the value obtainable by considering only the longer of the 2 delays. However, time diversity and combining do reduce $P_{O, E}$ and $P_{O, M}$, according to Equation $10 \mathrm{c}$, as Figure 8 shows, for the 2 delays $T_{1}=15$

TABLE 1 Parameters of the sites considered in the simulation of rain attenuation with the Synthetic Storm Technique (SST)

\begin{tabular}{|c|c|c|c|c|c|c|c|c|c|}
\hline Site & $\begin{array}{l}\text { Latitude, } \\
{ }^{\circ} \mathrm{N}\end{array}$ & $\begin{array}{l}\text { Longitude, } \\
{ }^{\circ} \mathrm{E}\end{array}$ & $\begin{array}{l}\text { Altitude, } \\
\text { m }\end{array}$ & $\begin{array}{l}\text { ITU-R } 0^{\circ} \mathrm{C} \\
\text { Height at the } \\
\text { Latitude, km }\end{array}$ & $\begin{array}{c}\text { SST } \\
\text { Rain + Melting } \\
\text { Layer Height, } \\
\text { km }\end{array}$ & $\begin{array}{l}\text { Mean Horizontal } \\
\text { Wind (Rain) } \\
\text { Speed, } \mathrm{m} / \mathrm{s}\end{array}$ & $\begin{array}{l}\text { Elevation } \\
\text { Angle, }^{\circ}\end{array}$ & $\begin{array}{l}\text { Frequency, } \\
\text { GHz }\end{array}$ & $\begin{array}{l}\text { Observation Period } \\
\text { and Size of Rain } \\
\text { Rate Database, y }\end{array}$ \\
\hline Tampa (Florida) & 27.60 & 277.7 & 15 & 4.182 & 4.567 & 8.3 & $52^{b}$ & 27.5 & $\begin{array}{c}1995-1998 \\
4\end{array}$ \\
\hline $\begin{array}{l}\text { Prague (Czech } \\
\text { Republic) }\end{array}$ & 50.1 & 14.5 & 254 & 2.689 & 2.835 & 10.6 & $31.7^{\mathrm{a}}$ & 19.7 & $\begin{array}{c}1999-2003> \\
4.2\end{array}$ \\
\hline
\end{tabular}

${ }^{\text {aALPHASAT. }}$

${ }^{\mathrm{b} A C T S}$.

'TDRS (Tracking and Data Relay Satellite).
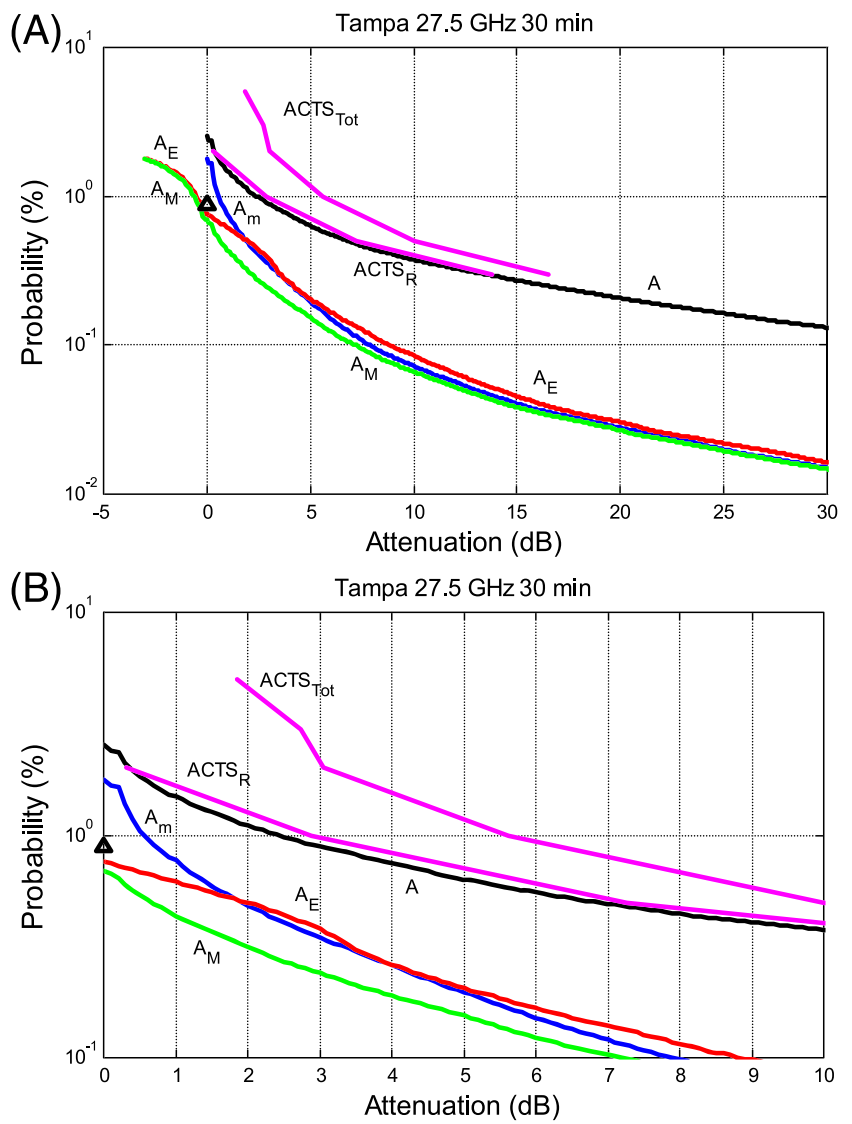

FIGURE 11 A, Complementary probability distribution functions, in an average year (\%), of $A$ (black), $A_{m}$ (blue), $A_{E}$ (red), and $A_{M}$ (green), at $27.5 \mathrm{GHz}$, for $T=30$ minutes, Tampa, $52^{\circ}$ slant path. In the range $-3,0 \mathrm{~dB}, A_{E}$ and $A_{M}$ are very similar. The triangle gives $P(A \geq 3)$, that is, the probability that the rain attenuation equal to the built-in power gain is exceeded in the direct link (black line). Synthetic Storm Technique. The curve labelled ACTS Tot is the experimental CDF of total attenuation. The curve labelled ACTS $\mathrm{R}$ is the estimated experimental CDF of rain attenuation. $B$, Detail of Figure 11A [Colour figure can be viewed at wileyonlinelibrary.com] 
and $T_{2}=30$ minutes. Now, the built-in power margin is $10 \times \log _{10} 3=4.8 \mathrm{~dB}$ and, accordingly, combining gives $P_{0, E}=P_{0, M}<P(A>4.8)<<P\left(A_{m}\right)$ $<P(A)$.

If the frequency is increased, eg, to the other ALPHASAT Aldo propagation experiment frequency, namely, $39.4 \mathrm{GHz}$, the general features of the results do not change. However, now, the combining is less useful because, as anticipated, the built-in power margin $G_{b}$ does not depend on frequency, as Figures 9 and 10 show.

\section{I TIME DIVERSITY RESULTS IN TAMPA, THE ISLE OF GUAM, AND PRAGUE ACCORDING TO THE SST}

The results reported above have shown general features that can also be found in other localities with different climate, such as Tampa, the Isle of Guam, and Prague (see Table 1 for geographical data and rain rate database).

Figure 11 shows the results for Tampa at $27.5 \mathrm{GHz}, 52^{\circ}$ elevation angle, relative to the ACTS link, both the ACTS experimental CDF ${ }^{27}$ and the SST predictions. Notice that the rain rate database used as input to the SST refers to 4 years, while the ACTS results refer to 5 years. ${ }^{30}$ The original experimental CDF of the ACTS measurements is relative to the total attenuation (labelled ACTS ), see Rogers and Crane. ${ }^{30}$ The CDF of rain attenuation only $\left(A C T S_{R}\right)$ is obtained from the former as follows. Because the fading due to the other tropospheric constituents, other than rain, is multiplicative in linear units and thus additive in $\mathrm{dB}$, the attenuation (in $\mathrm{dB}$ ) not due to rain is subtracted from the total attenuation $C D F$ at equal probability. Its value can found as follows. Because $P(R>0)=2.22 \%$ and $P(A>0)=2.52 \%$ (notice that $P(A>0)>P(R>0)$ always, see equation 18 of Matricciani ${ }^{23}$ ), the value to be subtracted is found at about $2.5 \%$, approximately $2.7 \mathrm{~dB}$, from the ACTS Turve. This value is practically constant for lower probabilities (see fig. 4 of Acosta et $\mathrm{al}^{31}$ ). The change in the phenomenon causing fading can be noticed very clearly in the change of the slope of the total attenuation CDF at about $2.5 \sim 3 \mathrm{~dB}$. The result of this shift to the left is the curve labelled ACTS $_{R}$, which agrees very well with the SST CDF, confirming, once again, the high reliability of this technique. As for the time diversity results, they are similar, in the general aspects, to those found for Spino d'Adda.

Figure 12 shows the results for Guam, $20.7 \mathrm{GHz}, 38^{\circ}$ slant path (see Acosta et al ${ }^{31}$ for details on the experimental data e SST test). Figure 13 shows the results for Prague in the slant path to ALPHASAT, at $19.7 \mathrm{GHz}, 31.7^{\circ}$.
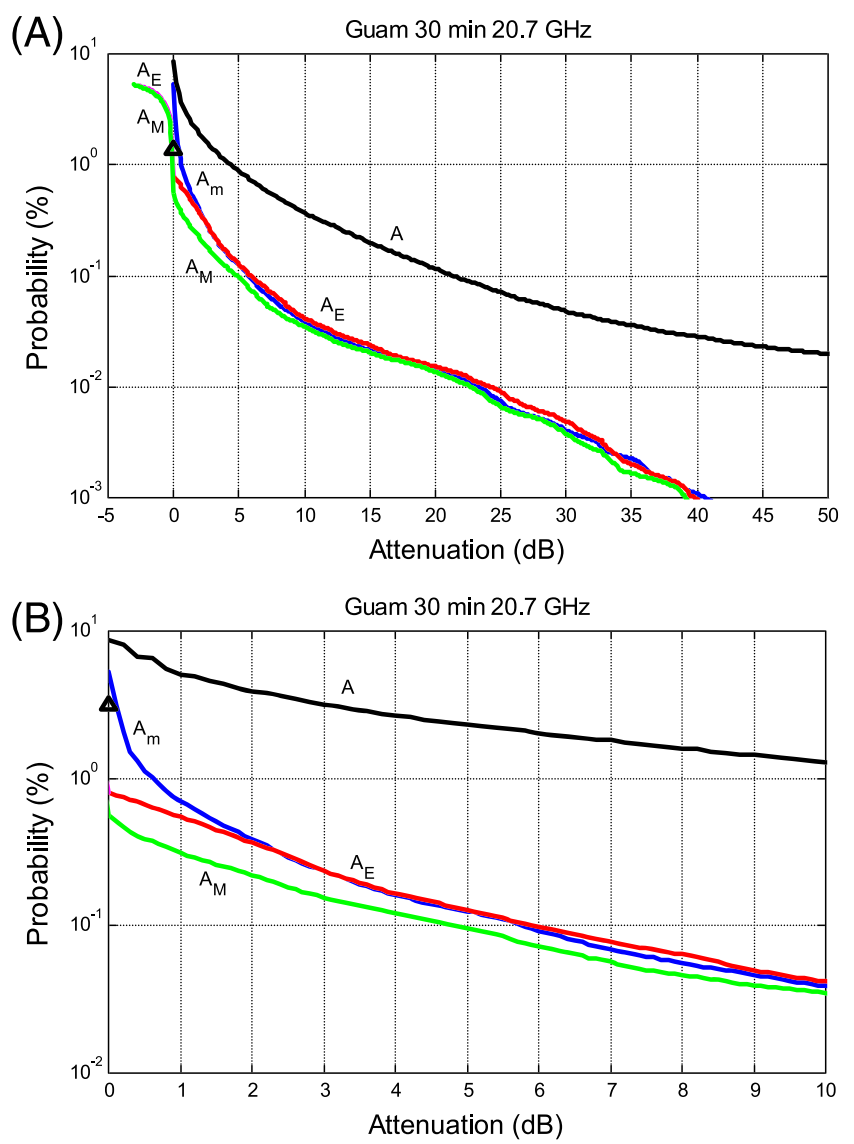

FIGURE 12 A, Complementary probability distribution functions, in an average year (\%), of $A$ (black), $A_{m}$ (blue), $A_{E}$ (red), and $A_{M}$ (green), at 20.7 GHz, for $T=30$ minutes, Guam, $38^{\circ}$ slant path. In the range $-3,0 \mathrm{~dB}, A_{E}$ and $A_{M}$ are very similar. The triangle gives $P(A \geq 3)$, that is, the probability that the rain attenuation equal to the built-in power gain is exceeded in the direct link (black line). Synthetic Storm Technique. B, Detail of Figure 12A [Colour figure can be viewed at wileyonlinelibrary.com] 

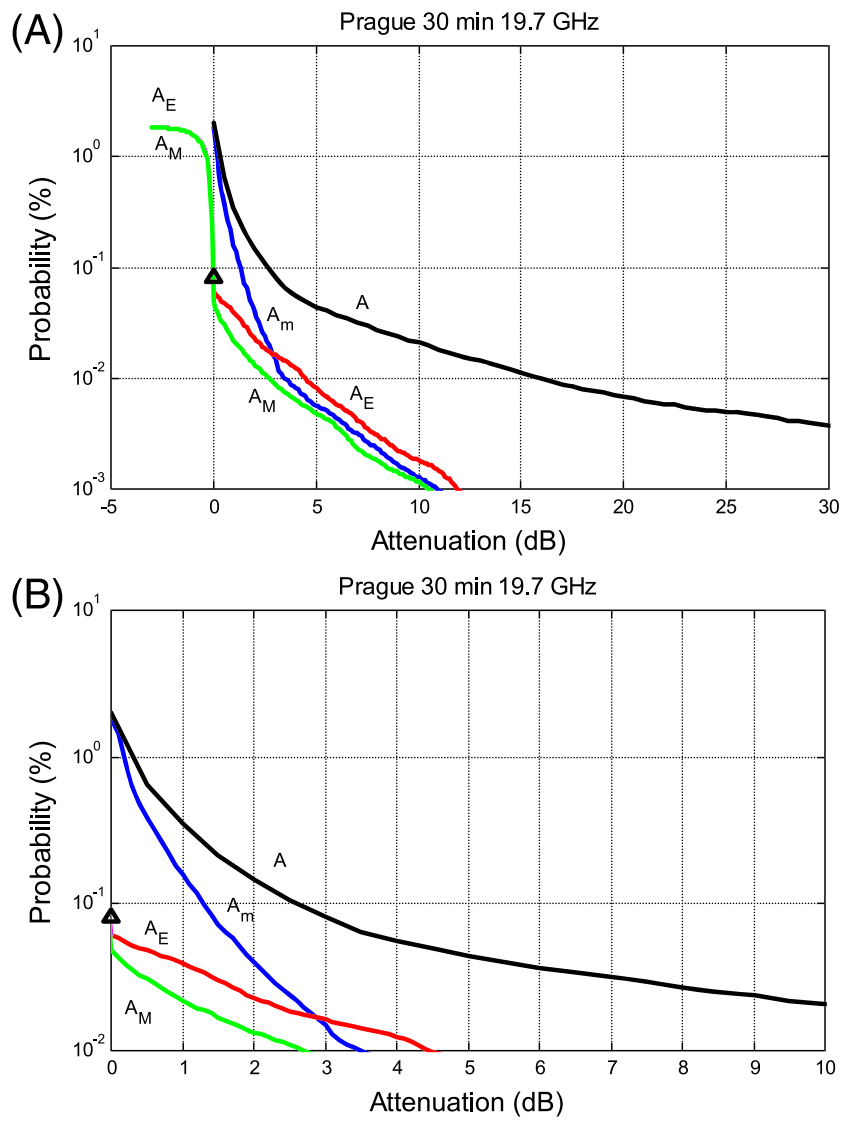

FIGURE 13 A, Complementary probability distribution functions, in an average year (\%), of $A$ (black), $A_{m}$ (blue), $A_{E}$ (red), and $A_{M}$ (green), at 19.7 GHz, for $T=30 \mathrm{~min}$, Prague, $31.7^{\circ}$ slant path. In the range $-3,0 \mathrm{~dB}, A_{E}$ and $A_{M}$ are very similar. The triangle gives $P(A \geq 3)$, that is, the probability that the rain attenuation equal to the built-in power gain is exceeded in the direct link (black line). Synthetic Storm Technique. B, Detail of Figure 13A [Colour figure can be viewed at wileyonlinelibrary.com]

In conclusion, we can see that the particular locality and its weather determine the diversity gain and the range in which the combining techniques can provide a sizable additive gain, not the general features.

\section{7 | REMARKS AND CONCLUSIONS}

Besides selection diversity, in this paper, the other 2 linear diversity techniques have also been studied, namely, the equal-gain combining and the maximal-ratio combining. The combining techniques can be applied both to time diversity, which uses 2 parallel channels, and to the classical ARQ techniques. Defined the instantaneous processing gain, and the equivalent attenuation in the 3 cases, we have shown examples of time series of the various parameters, based on the experimental rain attenuation time series recorded with the ITALSAT $18.7 \mathrm{GHz}$ beacon, in a $37.8^{\circ}$ slant path, at Spino d'Adda (Italy). Then, we have reported long term CDFs of the instantaneous gain and equivalent attenuation, by simulating rain attenuation time series at 19.7 and $39.4 \mathrm{GHz}$, path elevation angle $35.5^{\circ}$, with the SST, using on-site measured rain rate time series at Spino d'Adda for 10 years, in a slant path with ALPHASAT elevation angle.

The following time delays $T=1,5,10,20,30,60$ minutes have been studied. These delays can be reasonable for system application, but the actual value will depend on many compromises between the final user's necessities in the service area, or near-Earth and deep space communication distances, and the technology available. In system design, for example, there must be a trade-off between the time delay introduced in the link and the maximum fade tolerated, on the average, in the service area, in case of many ground stations. In general, the longer is the delay tolerated, the lower are the threshold and the link power margin needed, but the more demanding are hardware (memories) and software (signal processing) to be implemented, issues not studied. Results concerning Tampa, Guam, and Prague, as examples, show similar features.

In some occasions, it may be useful to give up time diversity and use the 2 parallel links directly by phasing the 2 radio frequency carriers at the transmitter. In this case, the equivalent transmitted power would increase by $6 \mathrm{~dB}$, while the receiver noise would be only that of the direct (ie, single) channel. The only CDF to be considered now is $P(A)$ and the new $P_{\Sigma}(A)$ is obtained from the previous figures by shifting the black curves to the left by $6 \mathrm{~dB}$, because this method can compensate fades up to $6 \mathrm{~dB}$ without any delay and diversity processing at the receiver, therefore $P_{\Sigma}(A>0)=P(A>6)$. This method could be used, of course, both in clear sky conditions and in rainy conditions. 
The additional gain that combining can give may seem small (less than $3 \mathrm{~dB}$ ) and expensive (more memory and processing), but we can appreciate its added value if we consider, for example, the large processing and hardware complexity that forward error-correcting codes require today to just gain few tenths of decibel, because they work close to Shannon limit.

The main conclusions we can draw from the results shown above are the following:

1. As expected, the instantaneous time diversity gain can be large when the delay time is long and rain attenuation is large.

2. Scintillation affects time diversity links as the direct links.

3. Defined the maximum fade duration $D_{S}(\mathrm{~min})$ at threshold $S(\mathrm{~dB})$, if the rain attenuation tolerated in the link budget must not exceed the link power margin $S(\mathrm{~dB})$ (ie, the fade margin), the time delay $T$ must satisfy the inequality $D_{S}<T$, therefore the minimum time delay is a function of the local fade duration maxima and power margin available. In a service area where sites experience different rain statistics, an area average should be considered, besides the technology issues mentioned (memories, processing, and service type).

4. Equal-gain and maximal-ratio combining can add up to $3 \mathrm{~dB}$ ( $4.8 \mathrm{~dB}$ for triple diversity) to the selection diversity gain (the simplest and more robust processing because it does not need to phase signals at the receiver), thus reducing the equivalent rain attenuation, just when the time diversity gain is negligible.

5. Equal-gain combining and maximal-ratio combining significantly reduce the fraction of time of rain attenuation in an average year to a value less than the probability of exceeding $3 \mathrm{~dB}$ (4.8 dB for triple diversity) in the direct link.

\section{ACKNOWLEDGEMENTS}

The author thanks the NASA Glenn Research Center (Cleveland, Ohio) for providing the rain rate data of Tampa and Guam and the Institute of Atmospheric Physics of Prague for providing the Prague rain rate data.

\section{REFERENCES}

1. Fukuchi H. Slant path attenuation at $20 \mathrm{GHz}$ for time diversity reception of future satellite broadcasting. Ursi F-open Symposium Colloque, Ravenscar, UK, 1992;6.5.1-6.5.4.

2. H. Fukuchi, Watson PA, Ismail AF. Proposal of novel attenuation mitigation technologies for future millimeter-wave satellite communications. Millennium Conference on Antennas and Propagation, Davos, Switzerland, 9-14 April 2000.

3. Ismail AF, Watson PA. Characteristics of fading and fade countermeasures on a satellite-Earth link operating in an equatorial climate, with reference to broadcast applications. IEE Proc Microwaves Antenn Propag. 2000;147:369-373.

4. Matricciani E. Time diversity as a rain attenuation countermeasure in satellite links in the $10-100 \mathrm{GHz}$ frequency bands. EuCAP 2006 , Nice, $6-10$ November, 2006.

5. Matricciani E. Time diversity in satellite links affected by rain: Prediction of the gain at different localities. EuCAP 2007, Edimburgh, 11-16 November, 2007.

6. Capsoni C, D'Amico M, Nebuloni R. Performance of time-diversity satellite communication systems investigated through radar simulation. The Second European Conference on Antennas and Propagation, EuCAP 2007, Edinburgh, 11-16 November 2007.

7. Pan QW, Allnutt JE, Tsui C. Evaluation of diversity and power control techniques for satellite communication systems in tropical and equatorial rain climates. IEEE Trans Antenn Propag. 2008;56:3293-3301.

8. Udofia KM, Otung IE. Evaluating time diversity performance on an on-board processing satellite to Earth station downlink. The Second International Conference on Next Generation Mobile Applications, Services, and Technologies, 2008, September 16-19, Cardiff, 325-330.

9. Fabbro V, Castanet L, Croce S, Riva C. Characterization and modelling of time diversity statistics for satellite communications from 12 to $50 \mathrm{GHz}$. Int J Satellite Comm. 2009;27:87-101.

10. Arapoglou PDM, Panagopoulos AD, Cottis PG. An analytical prediction model of time diversity performance for earth-space fade mitigation. Int J Antenn Propag. 2008;5

11. Capsoni C, D'Amico M, Nebuloni R. Radar simulation and physical modeling of time diversity satellite systems. Radio Sci. 2009;44. https://doi.org/ 10.1029/2009RS004142

12. Luini L, Capsoni C. Preliminary results from a physically-based methodology for the evaluation of a time diversity system. EuCAP 2011, 1-5, 11-15 April 2011, Rome, Italy.

13. Saad NWM, Ismail AF, Badron K, Isa F, Tharek AR, Din J. Proposed rain mitigation technique for Ka-band space-earth links in tropical climate. 2013 IEEE 11th Malaysia International Conference on Communications, November 26-28, Kuala Lumpur, 511-516.

14. Saad WNM, Ismail AF, Badron K, Sobli NHM. Assessments of time diversity rain fade mitigation technique for V-band space-earth link operating in tropical climate. Int J Electrical Energy. 2013;1. https://doi.org/10.12720/ijoee.1.4.268-273

15. Jong SL, D'Amico M, Lam HY. Performance of time diversity technique in heavy rain region. Proceedings of ISAP 2014, Kaohsiung, Taiwan, December 2-5.

16. Chodkaveekityada PC, Fukuchi H. Prediction model of time diversity using Japan rain radar data. Int J Satell Commun Networking. 2016; Published online in Wiley Online Library (wileyonlinelibrary.com). https://doi.org/10.1002/sat.1182

17. Chodkaveekityada PC, Fukuchi H. Time diversity evaluation for attenuation mitigation method using attenuation data in Thailand and Japan. Int J Satell Commun Networking. 2016; Published online in Wiley Online Library (wileyonlinelibrary.com). https://doi.org/10.1002/sat.1184 
18. Lin S, Costello DJ, Miller MJ. Automatic-repeat-request error control schemes. IEEE Commun Mag. 1984;22(12):5-17.

19. Pollara F, Ekroot L. Analysis of automatic repeat request methods for deep-space downlinks. The Telecommunications and Data Acquisition Progress Report, 1995, 42-122, Jet Propulsion Laboratory, 66-83.

20. Cheung KM. Problem formulation and analysis of the 1-hop ARQ links. InterPlanetary Progress Report, 2013, 42-194, Jet Propulsion Laboratory.

21. Uggalla L, Eastment J, Otung I. Mitigating the effects of rain-induced fading in satellite communications systems using time diversity in concert with maximal ratio combining. J Eng. 2016. https://doi.org/10.1049/joe.2015.0181

22. Brennan DG. Linear diversity combining techniques. Proc IRE. 1959;47:1075-1102.

23. Matricciani E. Physical-mathematical model of the dynamics of rain attenuation based on rain rate time series and two layer vertical structure of precipitation. Radio Sci. 1996;31:281-295.

24. Matricciani E. Micro scale site diversity in satellite and troposphere communication systems affected by rain attenuation. Space Commun. 2003;19:83-90.

25. Matricciani E, Riva C. $18.7 \mathrm{GHz}$ tropospheric scintillation and simultaneous rain attenuation measured at Spino d'Adda and Darmstadt with Italsat. Radio Sci. 2008;43. https://doi.org/10.1029/2007RS003688

26. Matricciani E, Riva C. The search for the most reliable long-term rain attenuation cdf of a slant path and the impact on prediction models. IEEE Trans Antenn Propag. 2005;53:3075-3079.

27. Matricciani E, Mauri M, Riva C. A rain rate data base useful to simulate reliable rain attenuation time series for applications to satellite and tropospheric communication systems. 2002 European Conference on Wireless Technology (ECWT 2002), Milan, 26-27 September 2002, $265-268$.

28. ITU-R rain height model for prediction methods, ITU-R P Series Recommendations-Radiowave Propagation 839-3, 2001, Geneva, Switzerland.

29. Matricciani E. Rain attenuation predicted with a two-layer rain model. Eur Trans Telecommun. 1991;2:715-727.

30. Rogers DV, Crane RK. Propagation results from the advanced communications technology satellite (ACTS) and related studies. Proceedings of ISAP2000, Fukuoka (Japan) August 2000, 9 pages.

31. Acosta R, Matricciani E, Riva C. Slant path attenuation and microscale site diversity gain measured and predicted in Guam with the synthetic storm technique at 20.7 GHz. EuCAP 2013, Gothenburg, 8-12 April, 2013, 1-4. 
APPENDIX A

\section{LIST OF MATHEMATICAL SYMBOLS AND THEIR DEFINITION}

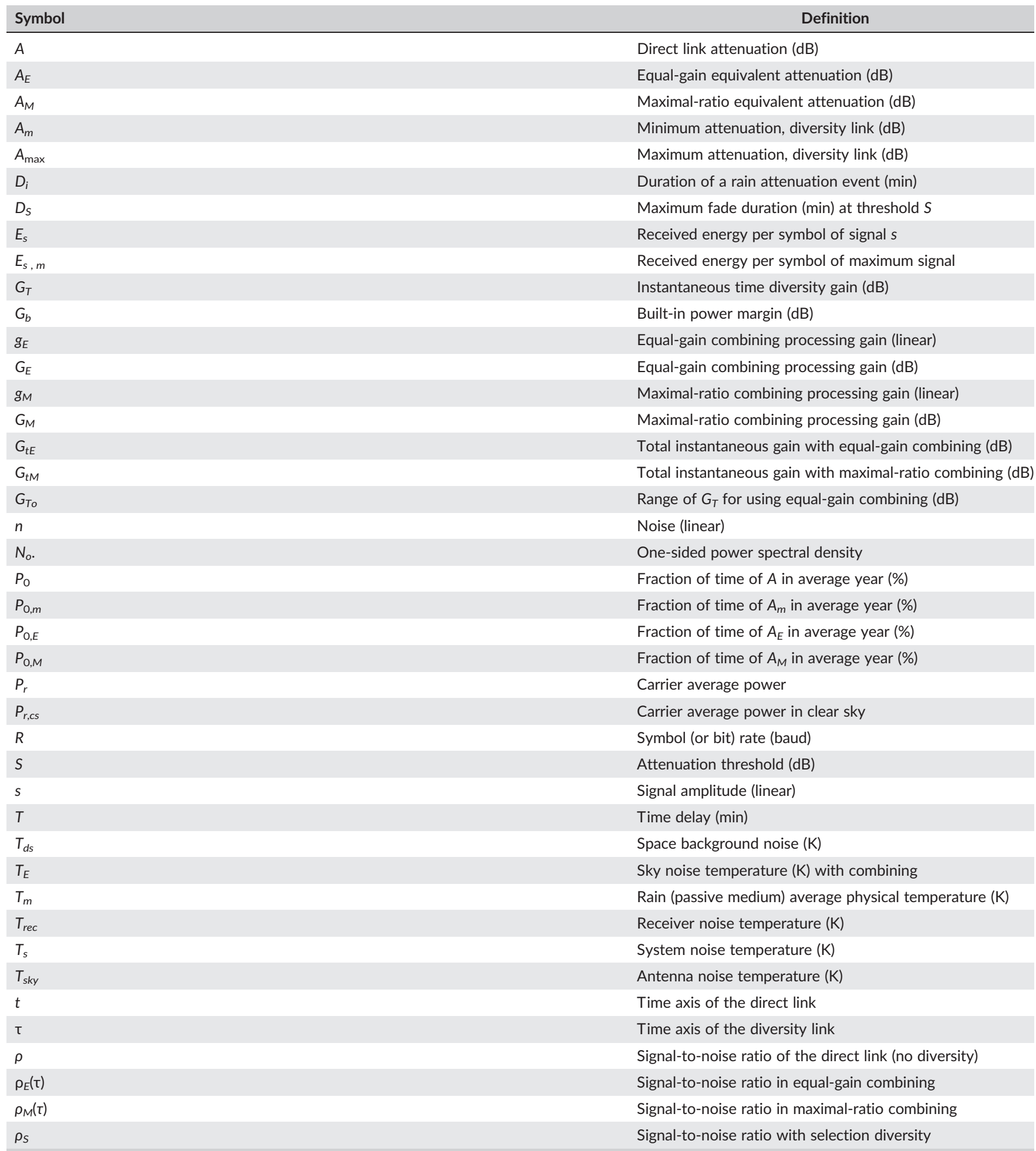




\section{APPENDIX B}

\section{NOISE}

The system (ie, total) noise is given by $T_{s}=T_{\text {sky }}+T_{\text {rec }}(\mathrm{K})$ where $T_{\text {rec }}(\mathrm{K})$ is the receiver noise temperature and $T_{\text {sky }}(\mathrm{K})$ is the antenna noise temperature. We are only interested in $T_{\text {sky }}$ because only this parameter is a function of rain attenuation. According to communication theory the probability of symbol, error does not depend on the instantaneous noise amplitude at the sampling time (see Equations $2 \mathrm{a}$ and $2 \mathrm{~b}$ ), but only on the noise power spectral density, therefore on the equivalent noise temperature. Therefore, in this appendix, we examine the impact of this parameter on the diversity link.

Because the rate of change of the baseband signal is much higher than that of rain attenuation or any other tropospheric fade, we can consider these equivalent noise temperatures as the instantaneous values for an interval long compared to data rate (eg, 1-s rain attenuation sampling time), without modifying in any significant way the analysis and conclusions.

$T_{s k y}$ is mainly caused by absorption, not by scattering phenomena. Raindrops, however, absorb and scatter electromagnetic waves, therefore rain attenuation is caused by both absorption and scattering. To be conservative, however, let us assume that $T_{\text {sky }}$ is caused by the total rain attenuation $A$ (dB), so that, by modelling rain attenuation as caused by an ideal passive medium with average physical temperature $T_{m}(K), \quad T_{s k y}$ is given by

$$
T_{s k y}=\frac{10^{\mathrm{A} / 10}-1}{10^{\mathrm{A} / 10}} T_{m}+\frac{1}{10^{\mathrm{A} / 10}} T_{d s},
$$

where $T_{d s}(\mathrm{~K})$ is the space background noise. Equation B1 is the $T_{s k y}$ standard model, with $T_{m} \approx 275 \mathrm{~K}$ usually assumed in communication link budgets, and it includes the possible noise increase due to water vapour, masked by $T_{m}$. Now, for the usual low values of $T_{d s}$ (approximately $3 \mathrm{~K}$ or few Kelvins) we can neglect the second term in Equation B1 and write

$$
T_{s k y} \approx \frac{10^{A / 10}-1}{10^{A / 10}} T_{m}<T_{m}
$$

In conclusion, $T_{s k y}, T_{s}$, and $N_{0}$ are function of $A(t)$ and thus of time, so that in time diversity, we should consider $N_{0}(t)$ and $N_{0}(t+T)=N_{0}(\tau)$. However, when $A(t) \approx A(t+T)=A(\tau), G_{T}(\tau) \approx 0 \mathrm{~dB}$, the processing gain $G_{E}(\tau)=G_{M}(\tau) \approx 3 \mathrm{~dB}$ (Section 3 ) and the noise power is constant, while when $A(t)$ and $A(\tau)$ are significantly different, $G_{E}(\tau)=G_{M}(\tau) \approx 0$ so that both equal-gain and maximal-ratio combining are not effective, and we use the direct link, not the diversity link. Therefore, the noise is that of the direct link. In between these 2 extremes, some dependency of noise on rain attenuation will remain, but this dependency does not impact on the results in the range of attenuation in which selection, equal-gain or maximal-ratio combining should be used.

This is clearly shown, for example, in Figure B1, T= 10 minutes, which reports the 2 direct link sky noise temperatures, given by Equation B2, applicable to selection diversity, and the total sky noise temperature $T_{E}$, namely, the sum of the 2 parallel link noise temperatures, applicable in equal-gain and maximal-ratio combining. It is evident, eg, in the interval between 6.0 and 6.4 hours, that when $G_{E}(\tau) \approx 3 \mathrm{~dB}$, and thus combining is most effective, the total sky noise temperature is practically constant, well less than $2 T_{m}$, in the example $T_{E} \approx 350 \sim 400 \mathrm{~K}$.
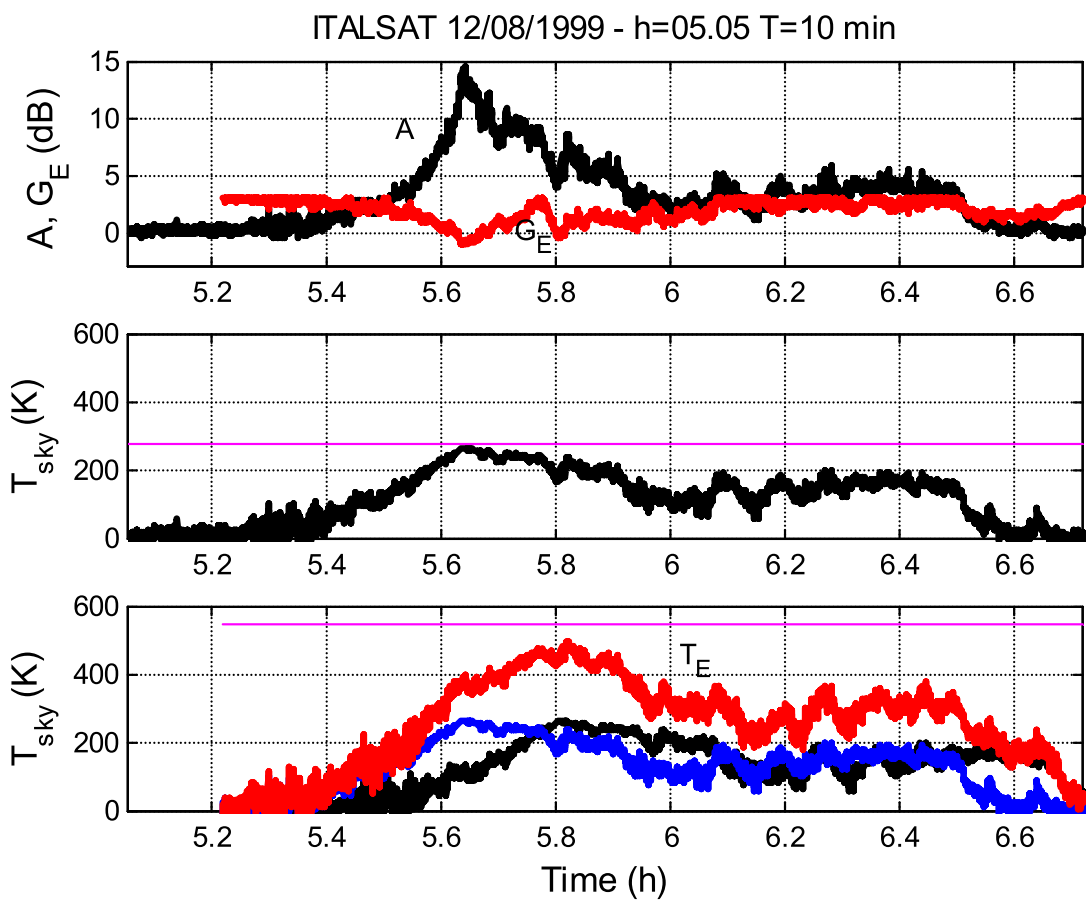

FIGURE B1 Example of rain attenuation and sky noise temperature time series with scintillation at $18.7 \mathrm{GHz}, 38.8^{\circ}$ slant path. $T=10$ minutes. Upper figure: $A(t)$ (black) and $G_{E}(\tau)($ red). Middle figure: single-link sky noise temperature. The horizontal (magenta) line gives $T_{S k y}=T_{m}$. Lower figure: sky noise temperature of the 2 parallel links (black and blue curves) and diversity link with combining $T_{E}$. The horizontal (magenta) line gives $T_{S k y}=2 T_{m}$. Local time [Colour figure can be viewed at wileyonlinelibrary.com] 


\section{APPENDIX C}

\section{SCINTILLATION AND COMBINING}

In equal-gain combining, the equivalent rain attenuation is given by Equation 9b:

$$
A_{E}=A_{m}-G_{E}=A_{m}-10 \times \log _{10}\left\{\frac{1}{2}\left(1+10^{-} \frac{G_{T}}{20}\right)^{2}\right\}
$$

By applying natural logarithms and considering that $\log (1+x) \leq x$, we can write Equation $\mathrm{C} 1$ as

$$
A_{E}=A_{m}+3-\frac{20}{\log 10} \times \log \left(1+10^{-\frac{G_{T}}{20}}\right) \leq A_{m}+3-8.68 \times 10^{-\frac{G_{T}}{20}}
$$

Now, since on the average

$$
G_{T}=A_{\max }-A_{m}=A_{\max , r}-A_{m, r}+\sqrt{\sigma_{\max }^{2}+\sigma_{m}^{2}}=G_{T, r}+\sigma_{c o m b},
$$

where the subscript $r$ explicitly indicates the values given only by rain (ie, filtered time series of fade), not scintillation, $\sigma_{\max }(\mathrm{dB}$ ) is the standard deviation of scintillation superposed to $A_{\max , r}, \sigma_{m}(\mathrm{~dB})$ is the standard deviation of the scintillation superposed to $A_{m, r}$ (both uncorrelated), and $\sigma_{\text {comb }}=\sqrt{\sigma_{\max }^{2}+\sigma_{m}^{2}}$ is the standard deviation of the total scintillation (all measured in the same interval as rain attenuation, eg, $1 \mathrm{~s}$ in this case) found with combining, we can write Equation C2 as

$$
A_{E} \leq A_{m, r}+\sigma+3-8.68 \times\left[10^{-\frac{\sigma_{T, r}}{20}} \times 10^{-\frac{\sigma_{c o m b}}{20}}\right]
$$

where $\sigma(\mathrm{dB})$ is the standard deviation of scintillation in the direct link. Standard deviation is a useful reference value for scintillation, although the results are general.

Expression C4 shows that the scintillation $\sigma_{\text {comb }}$ does not affect $\sigma$ but only the factor $10^{-} \frac{6_{T, r}}{20}$, which reduces the diversity gain. Even a very large $\sigma_{\text {comb }}=1 \mathrm{~dB}$ multiplies this factor only by0.89. A similar result holds also for maximal-ratio combining. In practice, any combining does not increase the scintillation of the direct link. The obvious reason for this result (found experimentally in the ITALSAT time series) is, of course, due to the fact that linear combining adds amplitudes, it does not multiply them: In other words, it does not add decibels. Notice that Equation C4 does not depend on elevation angle, therefore is applicable also when the scintillation of the direct link (namely, $\sigma$ ) is large, as in low elevation angle paths and electrically small antennas. 